\title{
Colposacropexia abdominal abierta con malla mixta en el prolapso de cúpula vaginal: experiencia en el Hospital de Quilpué, Chile
}

\author{
Francisco Kaplan D. ${ }^{1}$, Hernán Quinchavil A. ${ }^{1}$, Pablo Riera A. ${ }^{2}$, Robinson Flores . $^{1}$,

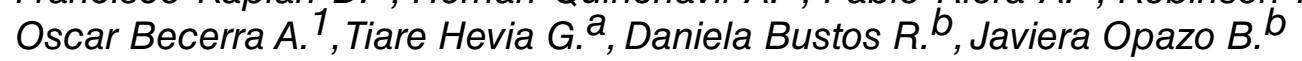 \\ ${ }^{1}$ Servicio de Obstetricia y Ginecología, ${ }^{2}$ Servicio de Urología, Hospital de Quilpué, Chile. \\ a Matrona, Policlínico de Ginecología, Hospital de Quilpué. b Alumnas, Escuela de Medicina, Universidad Andrés Bello.
}

\begin{abstract}
RESUMEN
Introducción: La colposacropexia por vía abdominal abierta (CSPA) es el gold standard para el tratamiento del prolapso de la cúpula vaginal (PCV). Objetivo: Mostrar nuestra experiencia, en un Hospital de mediana complejidad, en los primeros siete casos sometidos a un CSPA, reportando las complicaciones intra y post operatorias. Método: Se realizó un estudio prospectivo descriptivo en nuestras siete primeras pacientes portadoras de un prolapso de cúpula vaginal estadio III o IV (POP-Q) sintomáticas, sin incontinencia urinaria y sometidas a una CSPA, con una malla mixta en el Servicio de Obstetricia y Ginecología del Hospital de Quilpué. Resultados: Se reclutaron 7 pacientes. Ninguna de ellas presentaba complicaciones con riesgo vital peri-operatorio, en un período de seguimiento de 33 meses. En el 100\% de las pacientes se logró cura objetiva definida como un POP-Q estadio 0 o I, y buena calidad de vida según el Cuestionario de Impacto del Piso Pélvico (PFIQ-7, versión validada en español). Durante el seguimiento, sólo una paciente presentó exposición asintomática de $5 \mathrm{~mm}$ de la malla, resuelta con su resección por vía vaginal. Ninguna paciente requirió de una cirugía por prolapso de órganos pélvicos después de la CSPA. Conclusión: Los resultados obtenidos concuerdan con la literatura. La CSPA sigue siendo el gold standard para el tratamiento del PCV, no siendo superada por el momento, por ninguna otra técnica, incluso la colposacropexia laparoscópica. Estimamos que el uso de mallas parcialmente reabsorbibles pueden disminuir la exposición de mallas, sin embargo se requieren de más estudios.
\end{abstract}

\section{PALABRAS CLAVE: Colposacropexia, prolapso de cúpula vaginal, cirugía de prolapso genital}

\section{SUMMARY}

Introduction: The abdominal sacral-colpopexy is currently considered the gold standard for the management of (CSPA) vaginal vault prolapse (PCV). Objective: To report our surgical experience at a county hospital with our first seven abdominal sacral-colpopexy cases. Reporting intra and post-operative complications. Methods: We conducted a prospective descriptive study involving our first seven cases of patients with symptomatic vaginal vault prolapse stage III or IV (POP-Q) without urinary incontinence. All patients were undergoing CSPA with a partially absorbable mesh in the Obstetrics and Gynecology Department at the Quilpué Hospital. Results: Seven patients were recruited. None of them presented a life threatening complication during a mean follow up period of 33 months. A hundred percent of patients achieved objetive cure defined as POP-Q stage 0 or I and subjective cure defined as a significant improvement in a validated questionnaire (PFIQ-7 spanish version). During patients follow up, only one patient had an asymptomatic $5 \mathrm{~mm}$ mesh exposure, resolved with a vaginal resection. None of the patients required surgery for pelvic organ prolapse after the CSPA. Conclusion: These results are in agreement with the international literature. The CSPA continues to be the gold standard 
for the PCV treatment of vaginal vault prolapse and has not been surpassed by either vaginal technique or the laparoscopic sacral-colpopexy. We believe that the use of partially reabsorbable meshes can decrease the rate of mesh exposure, however further studies are required.

\section{KEY WORDS: Sacral-colpopexy, vaginal vault prolapsed, genital prolapsed surgery}

\section{INTRODUCCIÓN}

La disfunción del piso pélvico es una entidad clínica que involucra diversas condiciones: el prolapso de órganos pélvicos (POP), la incontinencia urinaria y anal, la disfunción sexual y el síndrome de dolor pelviano crónico, entre otros. EI POP es un problema frecuente y en aumento entre las mujeres. Se presenta entre el $30-50 \%$ de a lo largo de sus vidas y el $11 \%$ necesitará de una cirugía para su resolución. Cerca del $30 \%$ de las cirugías ginecológicas tendrán como indicación, alguna de las formas de disfunción del piso pélvico, constituyéndose en la indicación quirúrgica más frecuente en mujeres mayores de 70 años (1).

En los países desarrollados se le da gran importancia a este problema debido al efecto que tiene sobre la calidad de vida de las mujeres, por lo que se destina un importante porcentaje de los recursos económicos para su tratamiento y prevención, los que se estima aumentarán al doble en la próxima década debido al envejecimiento de la población, problema del cual nuestro país no está ajeno (2).

El prolapso de la cúpula vaginal (PCV) es una patología poco frecuente y de difícil manejo, que tiene una incidencia de $0,36-3,6$ por cada 1000 histerectomizadas (3).

En la actualidad es ampliamente aceptado que el adecuado soporte de la cúpula vaginal es un componente esencial para que la cirugía del POP sea efectiva y duradera en las mujeres portadoras de un POP avanzado. Por esta razón el diagnóstico preoperatorio del defecto apical es primordial en la evaluación del prolapso (4).

Existen muchos procedimientos que tratan de corregir el PCV pero hasta el momento ninguno ha superado a la colposacropexia abdominal abierta (CSPA), considerada como el gold standard, con tasas de éxito a largo plazo de $78-100 \%$ y tasas de satisfacción o eliminación completa de los síntomas de $85-100 \%$ (5).

El objetivo de este estudio es mostrar nuestra experiencia, en un Hospital de mediana complejidad, en el tratamiento quirúrgico de las primeras siete pacientes portadoras de un PCV en estadio III y IV sintomático, mediante una CSPA que incluye el tratamiento de la pared anterior y posterior de la vagina mediante el uso de una malla mixta parciamente reabsorbible.

\section{PACIENTES Y MÉTODO}

Se realizó un estudio prospectivo descriptivo en nuestras primeras 7 pacientes portadoras de un PCV estadio III o IV (POP-Q) sintomáticas sometidas a una CSPA con una malla mixta ( $50 \%$ polipropileno, no absorbible y $50 \%$ poliglecaprone, absorbible), monofilamento de baja densidad (Ultraproß) en el Servicio de Obstetricia y Ginecología del Hospital de Quilpué entre Septiembre de 2010 y Agosto de 2012.

Se seleccionaron pacientes sexualmente activas o aquellas que querían estarlo, pero que a causa del prolapso no lo estaban, con sus patologías de base compensadas, sin contraindicaciones quirúrgicas y con consentimiento informado. Se excluyeron aquellas pacientes con contraindicaciones médicas para cirugía, con algún tipo de incontinencia urinaria concomitante y aquellas que prefirieron la vía vaginal. Se les administró estrógenos locales bisemanal (Vacidox $\AA$ ) durante un mes pre operatorio y post operatorio a largo plazo. Se evaluaron síntomas de POP, POP-Q pre y post operatorio, exposiciones de malla y calidad de vida post operatoria. En todas las pacientes se usó profilaxis antibiótica con cefazolina $1 \mathrm{~g}$ y profilaxis de trombosis venosas con medias elásticas antitrombóticas pre y post operatorias, junto con heparina estándar subcutánea en el post operatorio.

La CSPA se basó en la técnica de Timmons para corregir el prolapso de cúpula y de la pared anterior y posterior, (Figura 1 a 7) (6). La malla en forma de $\mathrm{Y}$ invertida se ancló primero a la pared posterior, cúpula y pared anterior de la vagina y finalmente al promontorio (Figura 8 a 12). La malla anterior se ancló aproximadamente a $1 \mathrm{~cm}$ sobre el cuello vesical y la posterior a $2 \mathrm{~cm}$ sobre el músculo elevador del ano. Se utilizaron puntos no absorbibles de Polipropileno 2-0 (Prolene $₫$ ) para anclar los vértices inferiores de la malla a la vagina $\mathrm{y}$ al promontorio y puntos absorbibles de polidioxanona 2-0 (Monoplus $®$ ) en el resto de la pared vaginal aproximadamente 3 puntos por cada lado en la pared vaginal anterior, 4 puntos por cada lado en la pared vaginal posterior, dependiendo del largo de la vagina, y 3 puntos a la cúpula vaginal. La malla se dejó libre de tensión y se cubrió con peritoneo. Se utilizó un catéter vesical por 24 horas. Todas las pacientes fueron controladas a los 7 días y luego a los 3, 6, 12, 18 y 24 meses post operatorio. Se consideró falla objetiva a los estadios mayor 0 igual a II de POP. 


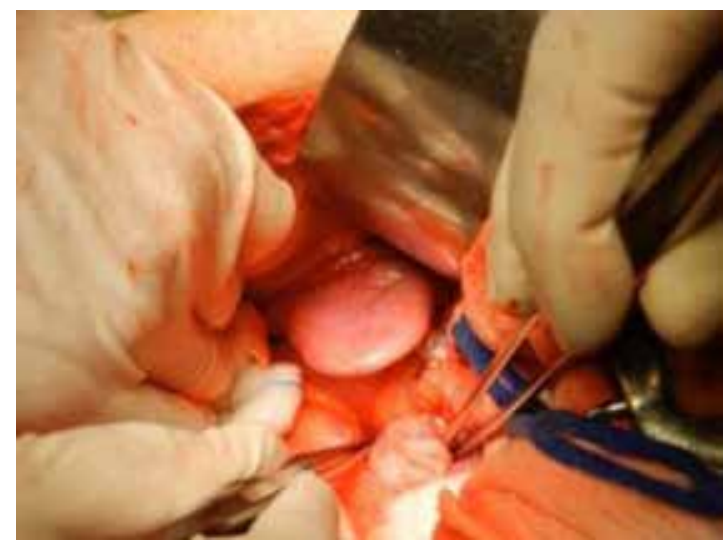

Figura 1. Cúpula vaginal.

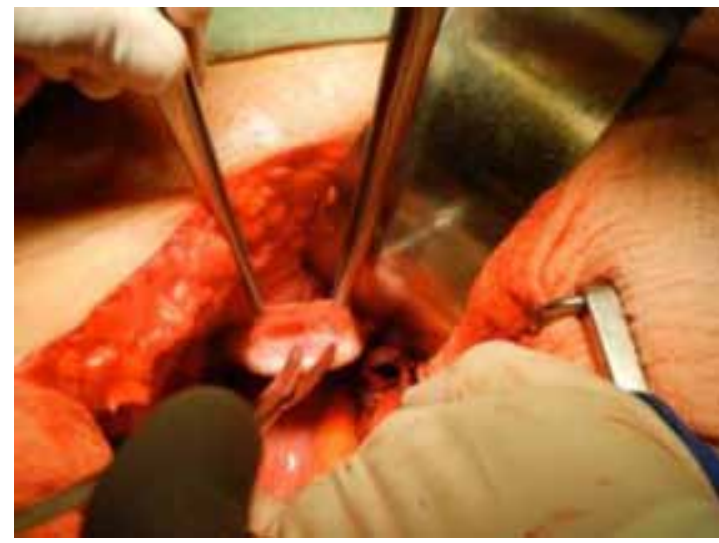

Figura 2. Apertura del peritoneo que recubre la cúpula vaginal y disección del espacio vesico vaginal.

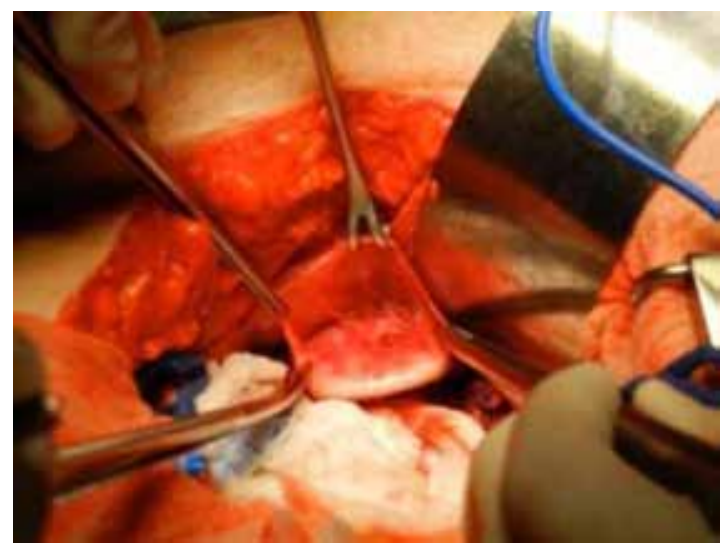

Figura 3. Disección del espacio vesico vaginal.

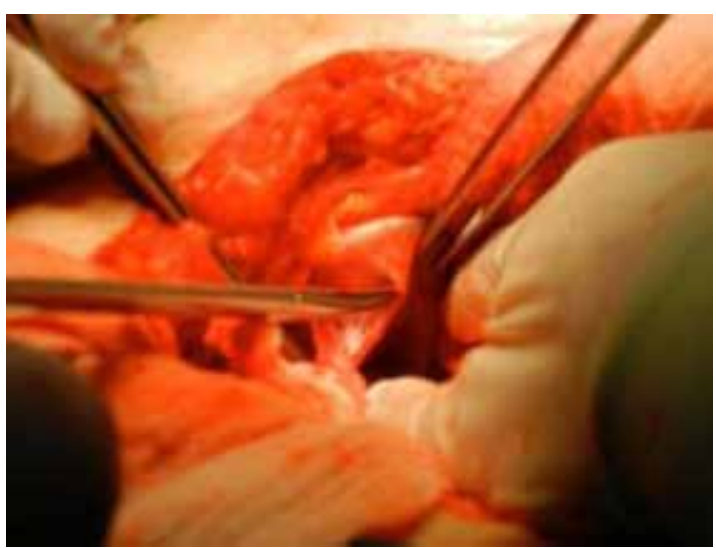

Figura 4. Disección del peritoneo de la pared vaginal posterior.

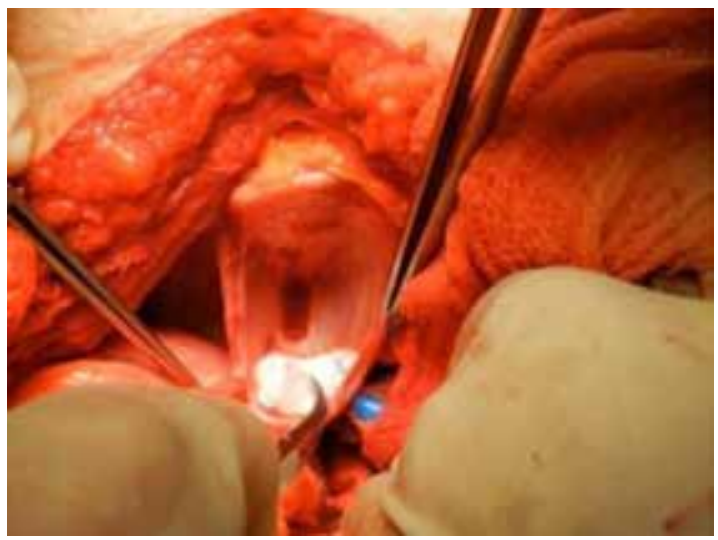

Figura 5. Disección del espacio recto vaginal.

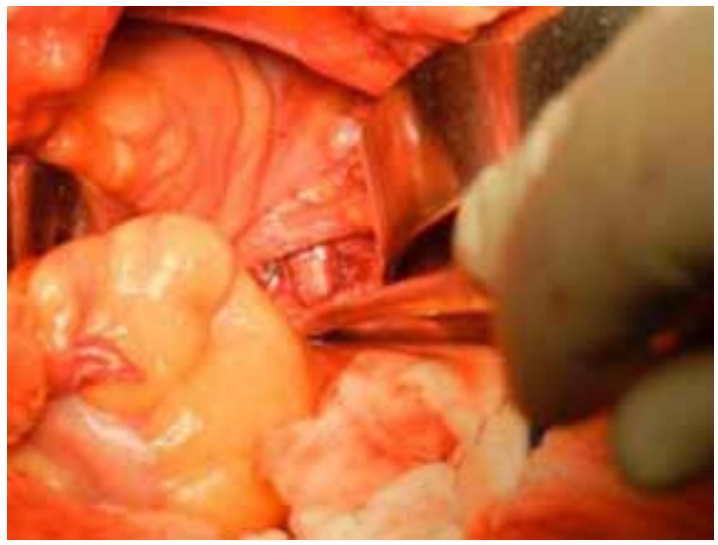

Figura 6. Ligamento longitudinal anterior. 


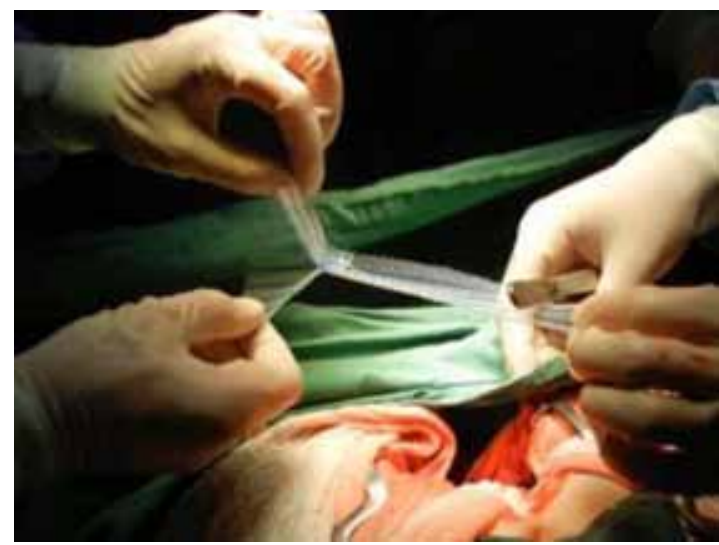

Figura 7. Malla Ultrapro® en forma de $\mathrm{Y}$ invertida.

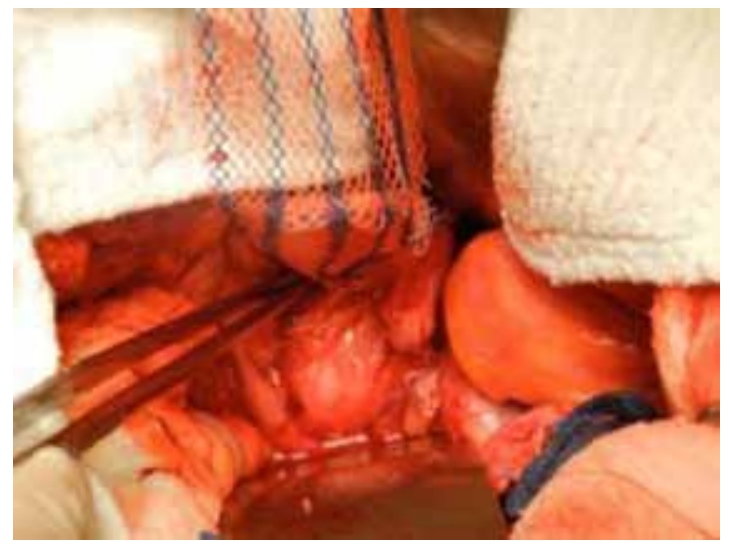

Figura 8. Malla anclada a la pared vaginal posterior.

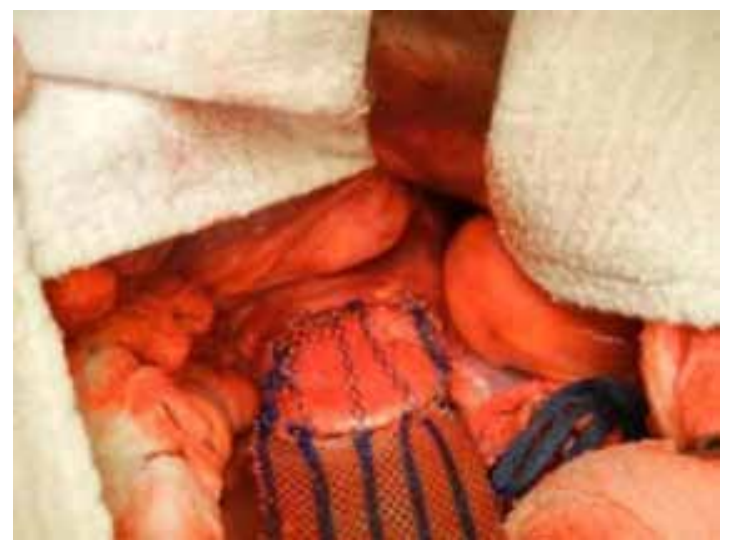

Figura 9. Malla anclada a la pared vaginal anterior.

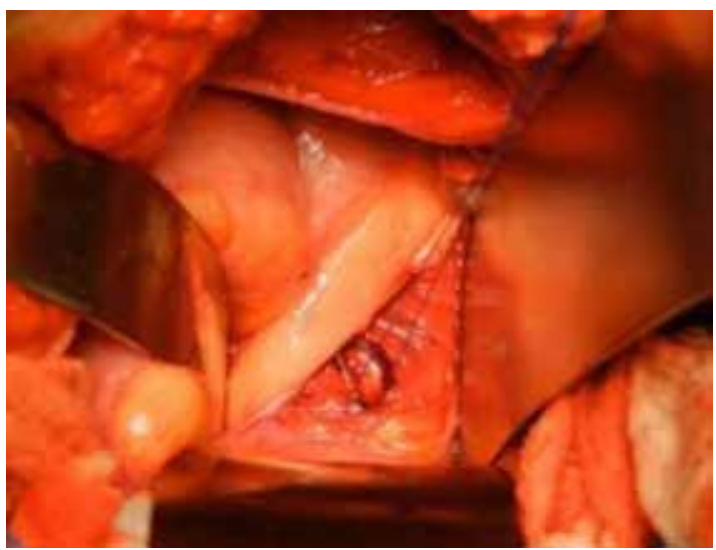

Figura 10. Ligamento longitudinal anterior con un punto de Prolene ${ }^{\circledR}$.

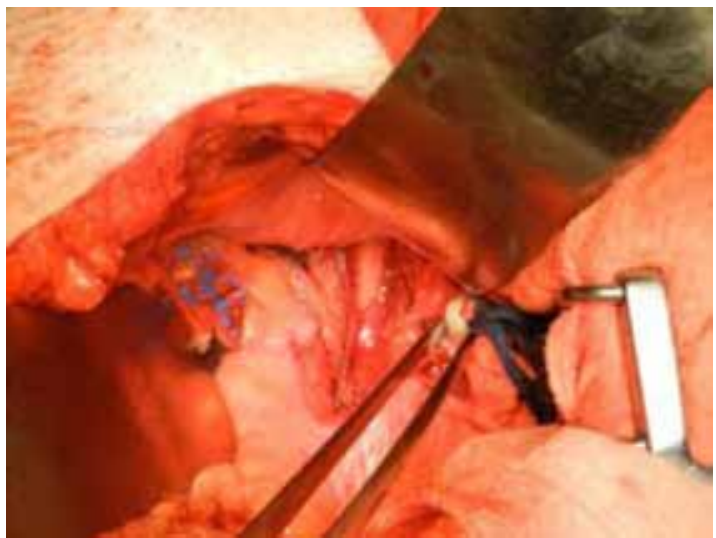

Figura 11. Malla anclada al ligamento longitudinal anterior.

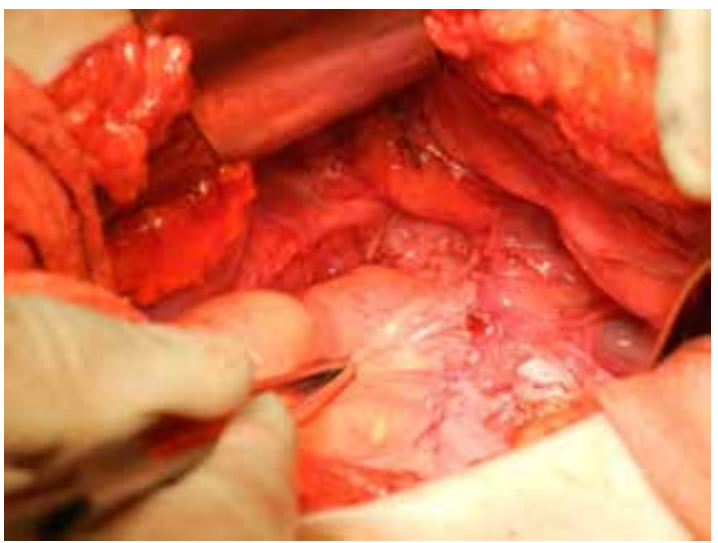

Figura 12. Malla peritonizada. 
Para evaluar calidad de vida post CSPA se utilizó el Cuestionario de Impacto del Piso Pélvico (PFIQ-7, versión validada en español) con un máximo de 63 puntos. Se consideró de 0-21 puntos mala calidad de vida, de 22-42 puntos regular calidad de vida y sobre 43 puntos buena calidad de vida (7).

\section{RESULTADOS}

El promedio de edad de las pacientes fue de $59 \pm 4,1$ años, índice de masa corporal (IMC) 27,2 y partos vaginales $3 \pm 1,15$. Cinco tenían antecedente de alguna cirugía correctiva de prolapso del compartimento anterior y/o posterior. Todas las pacientes presentaban síntomas de POP severos. Ninguna presentaba incontinencia urinaria de urgencia (IUU) ni incontinencia urinaria de esfuerzo (IUE).

El tiempo operatorio fue de $147 \pm 11,3$ minutos. No hubo complicaciones intra o post operatorias inmediatas. La estadía hospitalaria promedio fue de 3,1 \pm 1 día, y el tiempo promedio de seguimiento fue de $33 \pm 16$ meses. En un caso se abrió accidentalmente la vagina durante la cirugía. No hubo fallas objetivas del POP durante el período de seguimiento.

Sólo una paciente presentó a los 12 meses una exposición asintomática de la malla de $5 \mathrm{~mm}$, que correspondió a la paciente a la que accidentalmente se abrió la vagina durante la cirugía. La exposición de malla se resolvió mediante la resección quirúrgica de la zona expuesta en pabellón sin complicaciones.

Ninguna paciente presentó síntomas de bulto genital, algia pelviana, ni IUE de novo durante el seguimiento. Sólo una presentó IUU de novo y una paciente persistió con dispareunia pero de menor intensidad que antes de la cirugía. La calidad de vida y el impacto de la patología sobre sus vidas mejoró en todas las pacientes, evaluadas según encuesta PFIQ-7. No hubo infecciones intrahospitalarias. Ninguna paciente requirió de una cirugía por POP después de la CSPA al término de este estudio.

\section{DISCUSIÓN}

En nuestro estudio obtuvimos muy buenos resultados, con cura objetiva y subjetiva del $100 \%$ durante el período de seguimiento, lo cual es concordante con la literatura, y sin complicaciones intra o post operatorias importantes, gracias a la acuciosa selección de las pacientes, a pesar de que esto disminuyó considerablemente el número de casos, y a la preparación del equipo de cirujanos en la técnica quirúrgica.

El caso en el que hubo exposición asintomática de la malla de $5 \mathrm{~mm}$, fue pesquisado en un control de rutina a los 12 meses de seguimiento y coincidió con la paciente a la que se le abrió accidentalmente la vagina durante la cirugía, lo que refuerza la importancia de mantener intacta la pared vaginal, tal como se sugiere en la literatura.

La CSPA sigue siendo el gold estándar para el tratamiento de PCV a pesar que existe evidencia sobre la equivalencia con la colposacropexia laparoscópica (CSPL) para la resolución del defecto apical (8), sin embargo, un estudio sugiere que presentaría mayor tasa de reoperación del compartimento anterior, mayor tiempo operatorio, especialmente cuando no se cuenta con dispositivos de anclajes mecánicos de la malla al promontorio, aspecto muy relevante en hospitales como el nuestro debido a la alta demanda de cirugías y disponibilidad de pabellones (9).

En términos de alta precoz, retorno precoz a las actividades y la posibilidad de extender la malla más abajo en la pared posterior comparada con la cirugía abierta, Freeman y cols (8), no lograron demostrar ventajas de la vía laparoscópica sobre la abierta. Tampoco encontraron diferencias significativas en el dolor peri-operatorio entre ambas vías. Por lo tanto la CSPL se considera una excelente alternativa para el tratamiento de la PCV, sin embargo por el momento no es equivalente a la CSPA principalmente por su curva de aprendizaje.

La tasa de exposición de mallas en la CSPA es baja, especialmente si se utilizan mallas livianas de macroporo y en las que no se abre la vagina, como es el caso de la CSPA con histerectomía supra cervical (8). La mayoría de las mallas utilizadas en la CPSA se caracterizan por ser monofilamento, macroporo, de baja densidad y no reabsorbibles, confieren una resistencia hasta 10 veces superior a la máxima presión abdominal necesaria para romperlas, por lo que creemos, que el uso de una malla con similares características, pero con menor porcentaje de material no reabsorbible, tendría menos efectos adversos manteniendo los efectos favorables. Las mallas que cumplen con estos requisitos son las mixtas, en el caso particular de nuestro estudio utilizamos mallas Ultrapro®, que se caracterizan por ser parcialmente reabsorbible, con una reducción de hasta el $70 \%$ de cuerpo extraño implantado, sin perder la fuerza suficiente para soportar los tejidos (4 veces más que la presión abdominal máxima) permitiendo su óptima movilidad (10).

Por un tiempo se pensó que la cirugía de POP con mallas por vía vaginal desplazaría a la CSPA, en la actualidad las mallas vaginales han caído en desuso debido al alto riesgo de complicaciones (exposición, erosión, contractura, dispareunia y dolor pélvico crónico). Es así como en julio de 2011 la US Food and Drug Administration (FDA) realizó una alerta de seguridad acerca de las graves complicaciones asociadas a las mallas vaginales (11). Luego, en septiembre de 2011 las reclasificó 
de clase II (riesgo moderado) a clase III (alto riesgo), y en enero de 2012 la FDA ordenó realizar estudios de seguridad y eficacia post comercialización a los fabricantes de mallas vaginales, lo que llevó a la mayoría de los fabricantes a detener su producción. Actualmente la FDA solicitó a los fabricantes, datos clínicos que garanticen una seguridad y eficacia razonable de las mallas antes de su comercialización.

Si bien es cierto que la CSPA es el gold standard para el tratamiento del PCV, es necesario seleccionar muy bien a las pacientes, informarles sobre las alternativas terapéuticas ventajas y desventajas- y tener una formación quirúrgica adecuada para evitar las complicaciones potencialmente graves y resolverlas de la mejor forma cuando se presenten. Para esto es muy importante tener en cuenta la edad de la paciente, patologías concomitantes, cirugías de prolapso y abdominales previas, expectativas de las pacientes, especialmente si se mantienen sexualmente activas y la experiencia del cirujano.

Aunque la CPSA no presenta cura anatómica total a largo plazo (soporte anatómico perfecto) el $95 \%$ de las pacientes intervenidas no necesita una reintervención (12).

Por el momento, las distintas publicaciones relacionadas con la CSPA y la CSPL, no presentan una técnica estandarizada, existiendo series en que sólo reparan el nivel I $(5,8)$, hasta series en las que reparan todos los niveles, así como tampoco se usan mallas ni suturas del mismo tipo $(5,12)$. Además en la evaluación de los resultados tanto objetivos como subjetivos se utilizan distintos parámetros.

Será de gran utilidad realizar estudios prospectivos a largo plazo y con un poder adecuado que utilicen técnicas estandarizadas, mallas similares, los mismos parámetros de éxito objetivo y subjetivo junto con información de las complicaciones a corto y largo plazo para así tener una visión más cercana a la realidad de los resultados de las distintas técnicas tanto abiertas como laparoscópicas para luego poder compararlas.

\section{CONCLUSIÓN}

Los resultados obtenidos concuerdan con la literatura. La CSPA sigue siendo el gold standard para el tratamiento del PCV, no siendo superada por el momento, por ninguna otra técnica, incluso la colposacropexia laparoscópica. Estimamos que el uso de mallas parcialmente reabsorbibles pueden disminuir la exposición de mallas, sin embargo se requieren de más estudios.

\section{REFERENCIAS}

1. Olsen AL, Smith VJ, Bergstrom JO, Colling JC, Clark $A L$. Epidemiology of surgically managed pelvic organ prolapse and urinary incontinence. Obstet Gynecol 1997;89(4):501-6.

2. Cheon C, Maher C. Economics of pelvic organ prolapse surgery. Int Urogynecol J 2013;24(11):18736.

3. Dällenbach P, Kaelin-Gambirasio I, Jacob S, Dubuisson $\mathrm{JB}$, Boulvain $\mathrm{M}$. Incidence rate and risk factors for vaginal vault prolapse repair after hysterectomy. Int Urogynecol J Pelvic Floor Dysfunct 2008;19(12):16239.

4. Shull B, Bachofen C, Coates K, Kuehl T. A transvaginal approach to repair of apical and other associated sites of pelvic organ prolapse with uterosacral ligaments. Am J Obstet Gynecol 2000;183(6):1365-74.

5. Nygaard IE, McCreery R, Brubaker L, Connolly A, Cundiff G, Weber AM. Abdominal sacrocolpopexy: a comprehensive review. Obstet Gynecol 2004;104(4):805-23.

6. Timmons MC. Abdominal sacral colpopexy. In: Cardozo L, Staskin D (eds). Textbook of female urology and urogynecology. Isis Medical Media, London, 2001; pp 667-74.

7. Treszezamsky AD, Karp D, Dick-Biascoechea M, Ehsani N, Dancz C, Montoya TI, et al. Spanish translation and validation of four short pelvic floor disorders questionnaires. Int Urogynecol J 2013;24(4):655-70.

8. Freeman RM, Pantazis K, Thomson A, Frappell J, Bombieri L, Moran $\mathrm{P}$, et al. A randomised controlled trial of abdominal versus laparoscopic sacrocolpopexy for the treatment of post-hysterectomy vaginal vault prolapse: LAS study. Int Urogynecol J 2013; 24(3):377-84.

9. Khan A, Alperin M, Wu N, Clemens JQ, Dubina E, Pashos CL, Anger JT. Comparative outcomes of open versus laparoscopic sacrocolpopexy among medicare beneficiaries. Int Urogynecol J 2013;24(11):1883-91.

10. Doctor HG. Evaluation of various prosthetic materials and newer meshes for hernia repairs. J Minim Access Surg 2006;2(3):110-6.

11. FDA issues proposals to address risks associated with surgical mesh for transvaginal repair of pelvic organ prolapse. US Food and Drug Administration. Disponible en: http://www.fda.gov/NewsEvents/ Newsroom/PressAnnouncements/ucm395192.htm. Acceso el 29 de abril de 2014.

12. Nygaard I, Brubaker L, Zyczynski HM, Cundiff G, Richter $\mathrm{H}$, Gantz $\mathrm{M}$, et al. Long-term outcomes following abdominal sacrocolpopexy for pelvic organ prolapse. JAMA 2013; 309(19):2016-24. 